\title{
EAl Endorsed Transactions

\section{Can Be Applied for Bluetooth Low Energy to i-construction? : Characterization of BLE on Excavator}

\author{
Ryo Hamamoto ${ }^{1, *}$, Kazuomi Endo ${ }^{1}$, Hiroki Tanaka ${ }^{1}$, Koichi Yamashita ${ }^{1}$, Masayuki Kagoshima ${ }^{1}$, \\ Hiroshi Togo ${ }^{1}$, Kouta Mizukoshi ${ }^{2, \dagger}$, Hidenaga Kawaguchi ${ }^{3}$, and Yuta Kishino ${ }^{3}$
}

${ }^{1}$ Kobelco Construction Machinery Corporation Limited, Hiroshima, Japan.

${ }^{2}$ Fastics Corporation Limited, Tokyo, Japan.

${ }^{3}$ Nishiyama Corporation, Tokyo, Japan.

\begin{abstract}
The Government of Japan has declared that construction sites must implement $i$-construction, in which information communication technology (ICT) is used to improve construction site productivity and throughput. Examples of iconstruction include remote and automatic control systems for construction machinery, for which wireless communication is generally required; however, the wireless characteristics that must be considered in i-construction remain unclear. To clarify these characteristics, Bluetooth Low Energy (BLE) beacon module is implemented for construction machines, and we investigate its communication characteristics. We focussed on two evaluations: characterisation of the BLE unit module and its feasibility in i-construction. Experimental results were used to characterise BLE communication in a residential area and construction site. In the latter, BLE beacon module communication was possible between a sender located in an excavator attachment and a receiver in its cabin.
\end{abstract}

Keywords: Excavator, i-construction, Bluetooth, Wireless communication, Internet of Things

Received on 03 August 2020, accepted on 03 October 2020, published on 16 October 2020

Copyright (C) 2020 Ryo Hamamoto et al., licensed to EAI. This is an open access article distributed under the terms of the Creative Commons Attribution licence (http://creativecommons.org/licenses/by/3.0/), which permits unlimited use, distribution and reproduction in any medium so long as the original work is properly cited.

doi: 10.4108/eai.16-10-2020.166662

*Corresponding author. hamamoto.ryo@kobelco.com

$\dagger$ This work was conducted when he was working with Tokyo Musen Corporation, Tokyo, Japan

\section{Introduction}

An earlier version of this paper was presented at the 12 th International Workshop on Autonomous Self-Organizing Networks (ASON 2019) [1]. Please read the additional evaluations reported in the body of this paper.

How can the efficiency of labourers at a construction site be increased? To solve this issue, Japan is promoting work style reforms. In 2015, the Ministry of Land, Infrastructure, Transport, and Tourism of Japan declared that construction sites must drastically introduce information and communications technology (ICT) to improve productivity and throughput throughout the construction site [2]. This approach is called $i$ construction. In i-construction, many ICT technologies are applied to the operation of a construction site. Remote
[3] or automatic [4] control of construction machinery such as excavators, cranes, and other vehicles has been widely discussed. Automatic measurement of construction sites using drones is also another popular approach. Other i-construction applications include preventive maintenance [5] for construction machinery. In prevention maintenance, data gathered by various sensors installed on the construction machines are sent to servers via cellular or satellite networks. Each communication solution in i-construction is based on an Internet of Things (IoT) system. In IoT systems, networks comprise numerous data-gathering IoT devices (such as sensor devices), with each device sending data through wireless communication. Therefore, specific requirements specific to IT devices such as desktop PCs and servers must be met in order to implement these kinds of networks. For example, because IoT devices can be directly accessed by anyone at any time, additional security solutions must be 
introduced. Furthermore, maintenance methods of IoT devices are required for their effective operation.

Because IoT devices operate using battery power, one of their important requirements is power saving. If the batteries of several IoT devices are not charged, they cannot communicate to the network and preventing the IoT system to function properly. In order to meet this requirement, the use of a low-power-consumption communication protocol is crucial. To date, numerous low-power-consumption communication protocols for IoT systems have been proposed by many researchers and vendors. For example, LPWA (sigfox, LoRa, etc.) is a well-known communication protocol for IoT devices. Moreover, ZigBee and Wi-SAN have been also considered for application in IoT systems.

One of the standard wireless communication protocols for IoT systems is Bluetooth Low Energy (BLE) [6]. Because BLE is known for its low power consumption and its large communication range, a typical iconstruction implementation might include BLE communication modules. Various studies have evaluated the characteristics of BLE communication, but the required wireless communication characteristics for iconstruction are still unclear. In order to clarify this point, this paper examines the communication characteristics of BLE in consideration of the working area for different construction machines. In the evaluations, we used a BLE beacon module as the communication device. To develop the evaluation system, we used waterfall model. Experimental results that clarify the characteristics of communication in public roads and construction sites are provided. Moreover, assessments of a BLE beacon module in an excavator revealed that it was able to communicate between the sender in the excavator's attachment and the receiver in the cabin. Even if the size of the excavator was changed, communication could be established by adjusting the communication distance (Tx power). Finally, the results showed that wireless communication systems can be potentially applied for construction machinery.

\section{Contribution}

The contributions of this study are summarised as follows:

(i) The characteristics of BLE communication in public roads and construction sites were examined.

(ii) The communication characteristics of the beacon module in 13-, 20-, and 45-ton class excavators were assessed.

(iii) The relationship between the Received Signal Strength Indicator (RSSI) and communication availability using real machines was analysed.

\section{Organisation}

The rest of this paper is organised as follows. Section 2 describes the works related to this study. The experimental evaluation of BLE using a beacon module is explained and the results are discussed in Section 3. Section 4 describes the characteristics evaluation of BLE communication in construction excavators. Finally, Section 5 concludes this paper and explains future work.

\section{Related works}

This section summarises the works related to this study. In the area of Bluetooth communication, there have been many fundamental studies. For example, previous work [7] provides an overview of the communication and architecture of the Bluetooth framework. In addition, some protocols for operating Bluetooth are also summarised in this study. More recently, BLE has been proposed and evaluated for inter-vehicular communication [8]. From this work, BLE can be used to form the infrastructure of Vehicle-to-everything (V2X) systems. BLE can be used to construct not only a singlehop network (in which the sender and receiver communicate directly) but also multi-hop networks (in which the sender and receiver communicate via multiple terminals). The performance of Bluetooth-based multihop networks was evaluated in [9]. From the findings of this study, Bluetooth-using terminals were found to be able to communicate over long distances via relay terminals. The studies discussed above demonstrated that Bluetooth is a very useful communication protocol. In addition, the impact of Bluetooth communication broadcast has also been investigated [10]. This study shows that careful network design is necessary. Some studies have evaluated the Bluetooth communication characteristics considering an IEEE 802.11 and a ZigBee coexistence environment [11, 12]. However, previous studies do not focus the communication characteristics considering construction machines such as excavators. This study evaluates it using real machines. The results of this paper are useful to design the application for construction machines using BLE.

Applications of Bluetooth communications have been described in many papers. For example, [13] describes a position prediction method using BLE. In [14], a system for multiple device control was implemented using Bluetooth. Actuator control using Bluetooth communication is evaluated in [15] and a manufacturing network application using Bluetooth is discussed in [16]. Although much research effort has focussed on the topic of Bluetooth, the literature on the detailed evaluation of Bluetooth communication characteristics is still insufficient. In particular, there have been fewer experimental evaluations of the use of Bluetooth in construction machinery than on the use of Wi-Fi or WiMAX [17]. Thus, the evaluation results described in this paper can be utilised as implementation guidelines in i-construction. In the next section, experimental evaluations of BLE communication in two specific environments are presented. The characterisation was conducted using a BLE communication module installed in different excavators. Note that this paper is not 


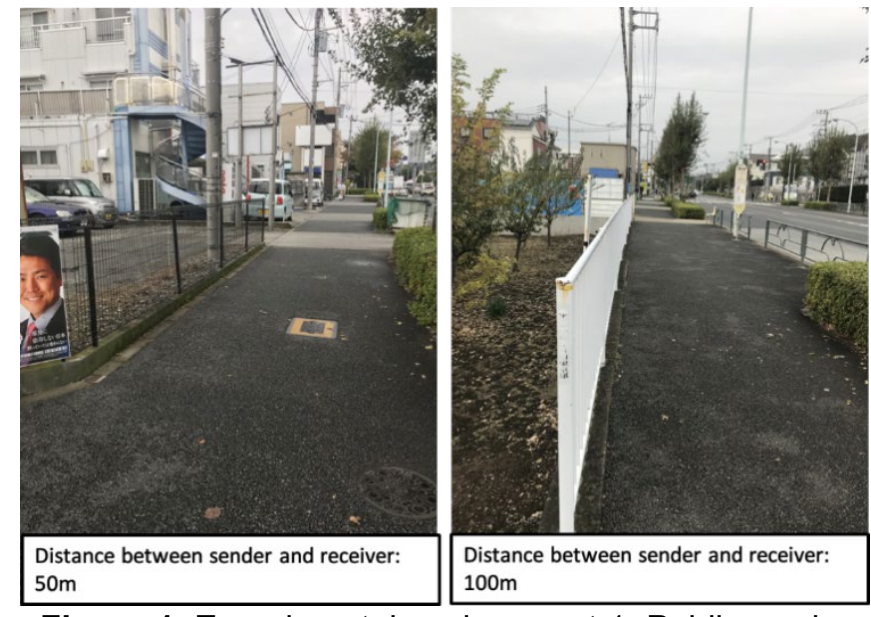

Figure 1. Experimental environment 1: Public road.

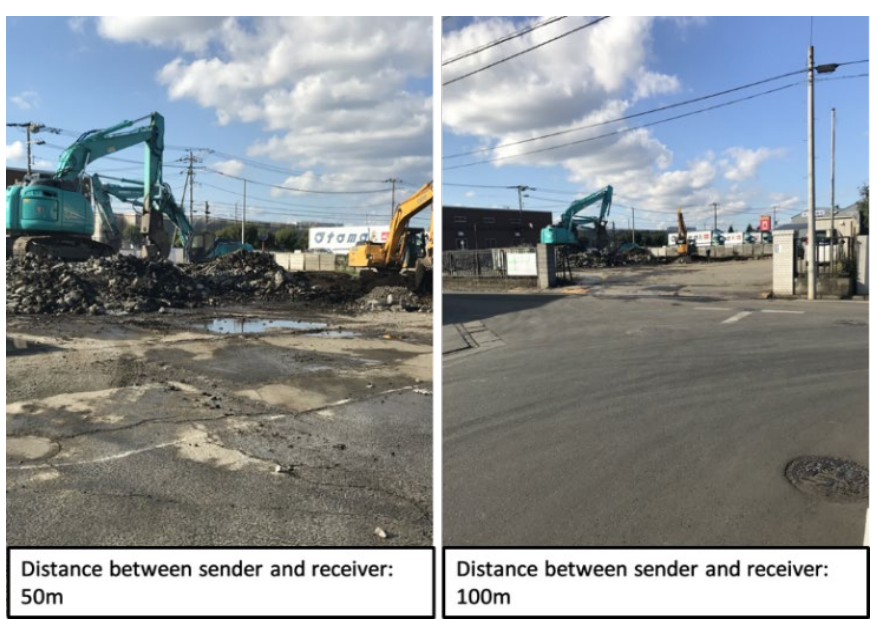

Figure 2. Experimental environment 2: Construction site.

proposing the application using BLE considering construction machines. In future, we will propose some applications based on this paper's results.

\section{Evaluation of Bluetooth Low Energy in Various Situations}

This section shows the experimental evaluation using a Bluetooth Low Energy beacon module. Specifically, we focused on two environments: a public road and a construction site. Note that, because this section reports on a basic version of the study described in the following section, we describe a simple experimental set up. Moreover, we used black box test for testing approach.

\subsection{Experimental Settings for Unit Test}

The sender was a beacon module using BLE 4.2 with a communication distance (Tx power) of $100 \mathrm{~m}$. The receiver was an iPad mini. Two points were evaluated:
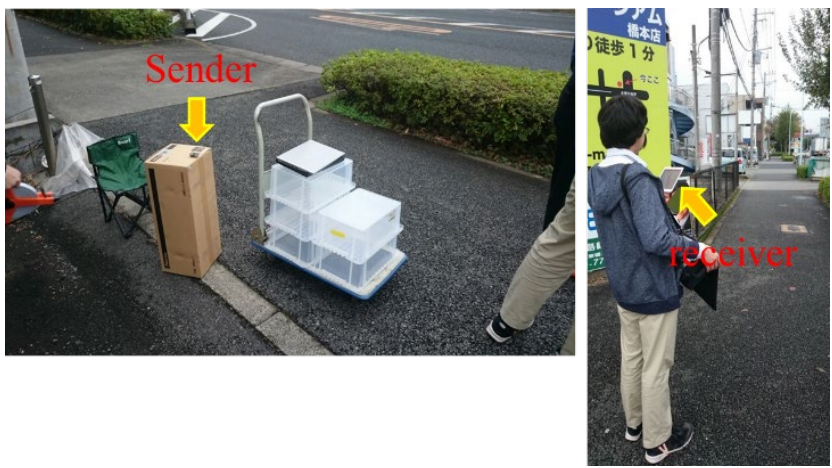

Figure 3. Sender and receiver.

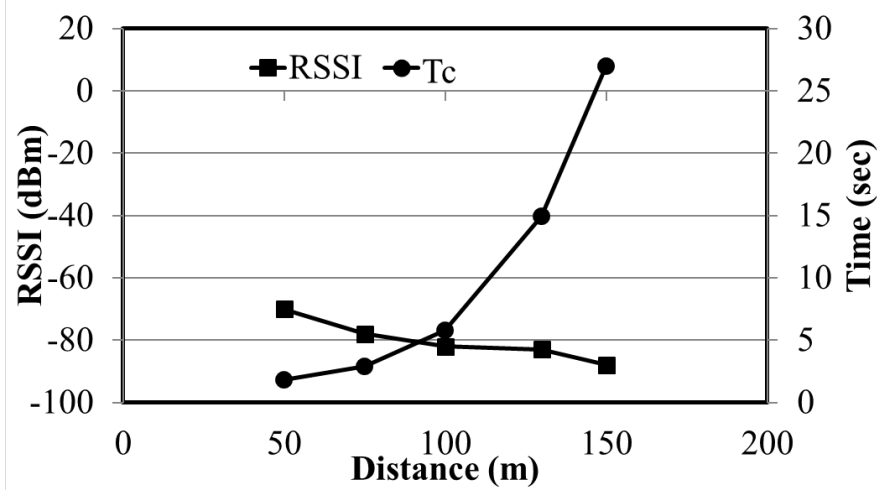

Figure 4. Relationship among RSSI, $T_{c}$, and communication distance at experimental environment 1.

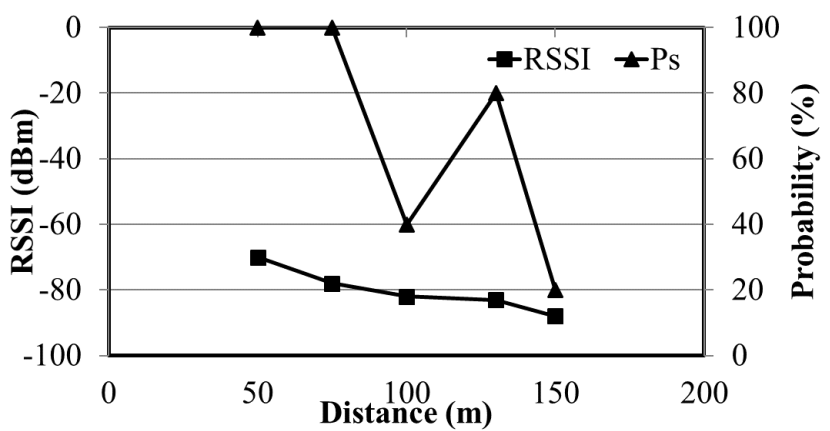

Figure 5. Relationship among RSSI, $P_{s}$, and communication distance in experimental environment 1.

- The relationship among the distance between the sender and the receiver, the RSSI, and the communication establishment time $\left(T_{c}\right)$

- The relationship among the distance between the sender and the receiver, the RSSI, and communication success percentage $\left(P_{s}\right)$

$T_{c}$ is the time difference between the start of beacon transmission and the completion of reception. $P_{s}$ is the percentage of beacon signals received for the trials at each distance. As this study represented the first step in assessing the applicability of BLE for i-construction, we used a simple evaluation item. In the future, we will carry out evaluations for BLE using more detailed and complex 


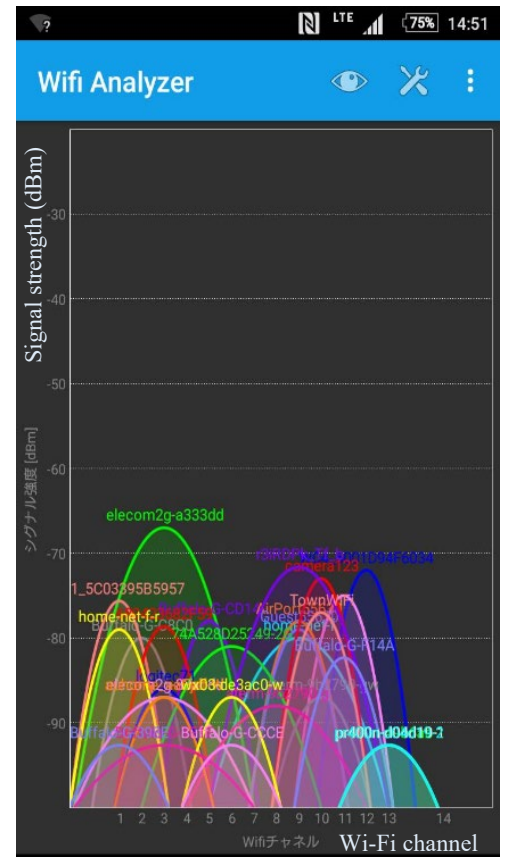

Figure 6. Utilisation of the 2.4-GHz band when the distance is $100 \mathrm{~m}$ (public road). Horizontal axis and vertical axis mean Wi-Fi channel and signal strength, respectively.

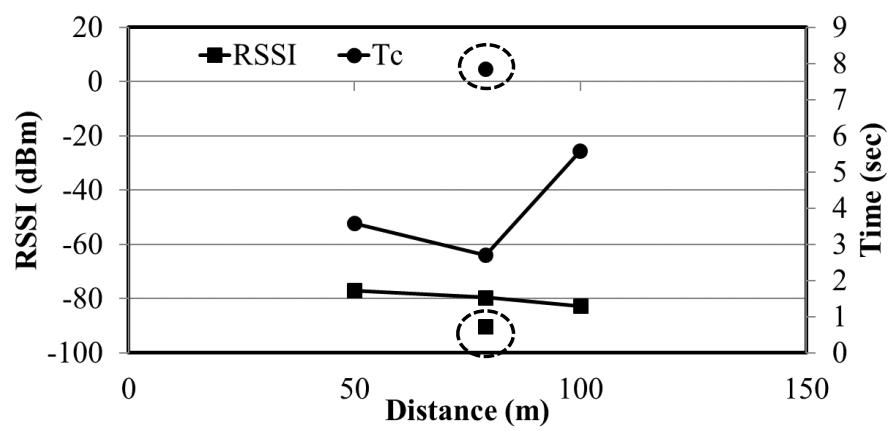

Figure 7. Relationship among RSSI, $T_{c}$, and communication distance in experimental environment 2.

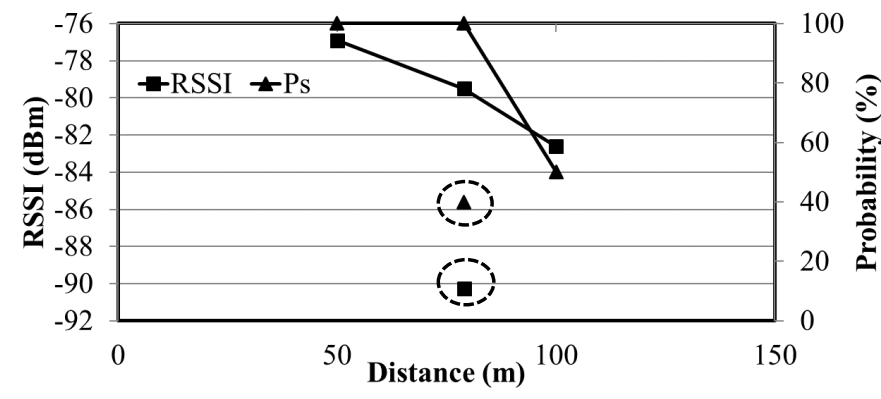

Figure 8. Relationship among RSSI, $P_{s}$, and communication distance in experimental environment 2.

characteristics. The evaluations were carried out at a

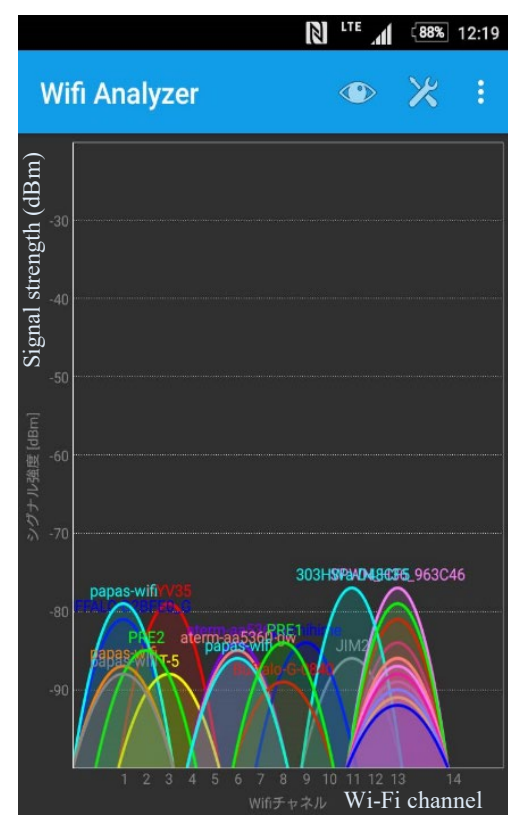

Figure 9. Utilisation of the 2.4-GHz band when the distance is $80 \mathrm{~m}$ (construction site). Horizontal axis and vertical axis mean Wi-Fi channel and signal strength, respectively.

public road (Fig. 1) in Chofu, Tokyo and at a construction site (Fig. 2) in Sagamihara, Kanagawa. Heavy vehicular traffic was observed in the public road and some excavators were working at the construction site, indicating that the network environments of both areas changed dynamically. However, the trends were nearly the same at the two sites. The beacon module was fixed and the iPad (Fig. 3) was mobile. The RSSI, $T_{c}$, and $P_{s}$ were measured over a distance from 50 to $150 \mathrm{~m}$. At each distance, 10 trials were conducted and the average was recorded as the result. The payload size was 70 bytes. The BLE communication mode was set as connection. The total measurement time was $60 \mathrm{~s}$. The evaluation system's algorithm is summarized in the Appendix A (see Algorithm 1 and Algorithm 2).

\subsection{Experimental results and discussion in each situation}

First, we show the results obtained at the public road. Fig. 4 shows the relationship among communication distance, RSSI, and $T_{c}$. Additionally, Fig. 5 plots the relationship among communication distance, RSSI, and $P_{s}$. As shown in Fig. 4, $T_{c}$ increased when the distance increased. Furthermore, RSSI decreased when the distance increased. As shown in Fig. 5, as the distance increased, $P_{s}$ showed a decreasing trend. These results indicate that a decrease in radio field intensity affects the communication establishment time and hence the success rate of the communication. The maximum difference of $T_{c}$ was $25 \mathrm{~s}$. In Fig. 5, the percentage appears to decrease significantly 


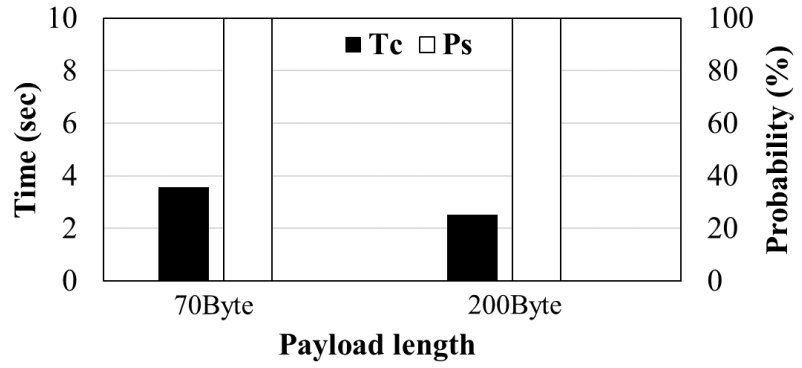

Figure 10. Relationship among payload size, $T_{c}$, and $P_{s}$ when communication distance equals to 50 $\mathrm{m}$ (communication in prospect).

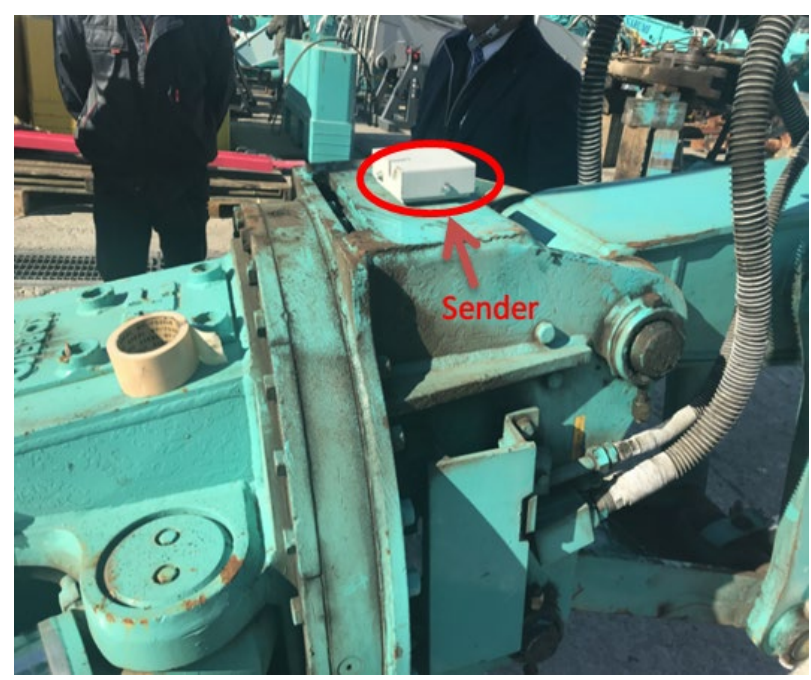

Figure 11. Position of the sender (in SK-470-10). The others machine set the sender in the same position.

$(40 \%)$ when the distance reaches $100 \mathrm{~m}$. The reason for this is the interference of other radio waves. Fig. 6 shows the utilisation of the $2.4-\mathrm{GHz}$ band at a distance of $100 \mathrm{~m}$ (by Wi-Fi analyser [18]). It is seen from the results that many radio wave generators were present. In this experimental location, there were many residences near the area that probably have Wi-Fi access points, a factor that appears to have adversely affected the results.

Second, we show the results obtained at the construction site. Fig. 7 describes the relationship among the communication distance, the RSSI, and $T_{c}$; Fig. 8 shows the relationship among the communication distance, the RSSI, and $P_{s}$. In this evaluation, results were measured at distances of 50, 80, and $100 \mathrm{~m}$. In Figs. 7 and 8 , the dotted black circles indicate the results of communication outside prospect, in which the excavator serves as an obstruction. As shown in Fig. 7, the difference between the in- and out-of-prospect $T_{c}$ is $7 \mathrm{~s}$, indicating that the communication establishment time is strongly affected by the obstruction. The reason for this is the decrease in RSSI introduced by the obstruction. It is difficult to implement stable communication at this RSSI (approximately -90 dBm). As shown in Fig. 8, the outside prospect communication results differ significantly from

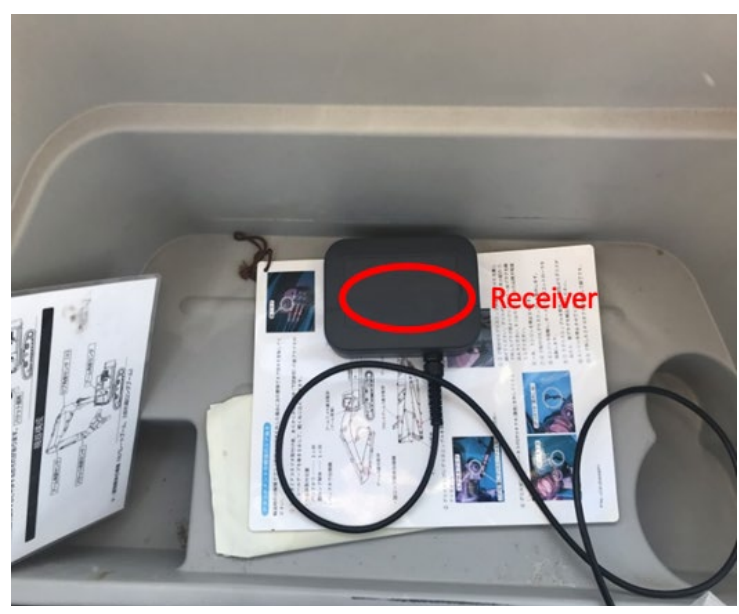

Figure 12. Position of the receiver in the excavator Cabin.

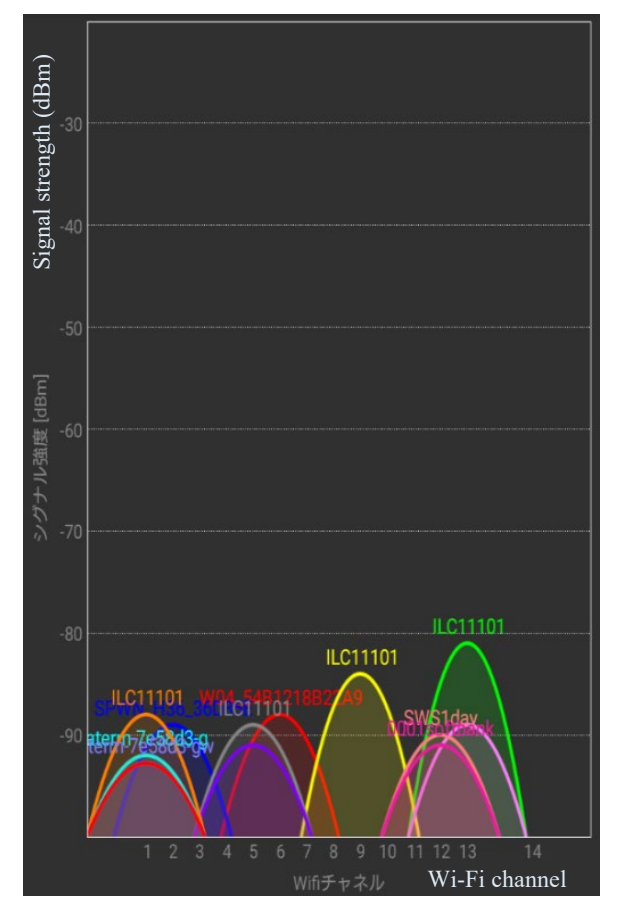

Figure 13. Utilisation of the $2.4-\mathrm{GHz}$ band in the evaluation field. Horizontal axis and vertical axis mean Wi-Fi channel and signal strength, respectively.

the in-prospect results; in terms of $P_{s}$, the results differ by $60 \%$. From these results, we can conclude that obstructions must be considered when implementing a BLE network. Fig. 9 shows the utilisation of the $2.4-\mathrm{GHz}$ band at a distance of $80 \mathrm{~m}$. It is seen that, although the utilisation trend is the same as in the public road (Fig. 6), the number of radio wave generators is reduced. The reason for this is that the experimental area was surrounded by an industrial park; as a result, there were few Wi-Fi access points and the distances between the radio wave generator and beacon module or iPad were 
smaller than in the public road case. Finally, we evaluated the effect of the payload size of the $T_{c}$ and $P_{s}$. Fig. 10 shows the relationship among payload size, $T_{c}$, and $P_{s}$ at a distance of $50 \mathrm{~m}$ in construction site. The results show the communication in the prospect case. As shown in the figure, the difference in $T_{c}$ is approximately $1 \mathrm{~s}$ and $P_{s}$ is $100 \%$ at each payload size. Therefore, in this evaluation the payload size did not have a significant impact on the BLE communication performance.

\section{Evaluation of Bluetooth Low Energy using Excavators}

This section describes an experimental evaluation using a BLE beacon module installed in several excavators. We used three different excavator classes. Note that, the testing approach is same as Section 3. Same as Section 3, furthermore, the evaluation system's algorithm is summarized in the Appendix A (see Algorithm1 and Algorithm 3).

\subsection{Experimental Settings for Unit Test}

In the evaluations, a beacon module using BLE 4.2 was

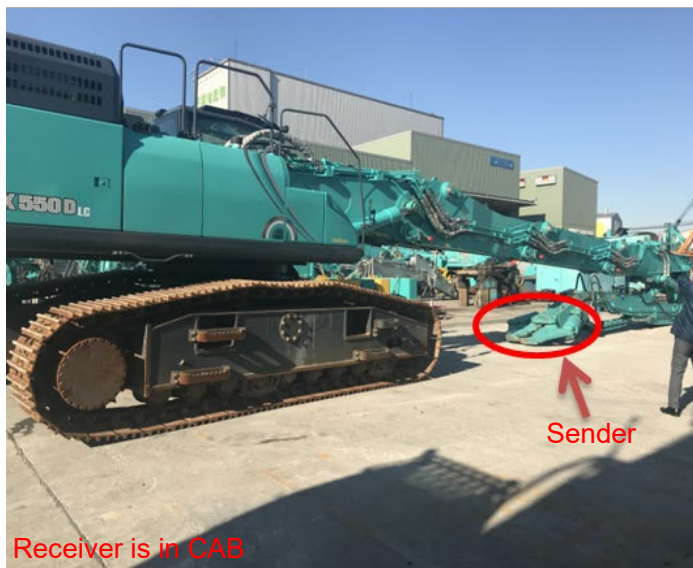

Parking

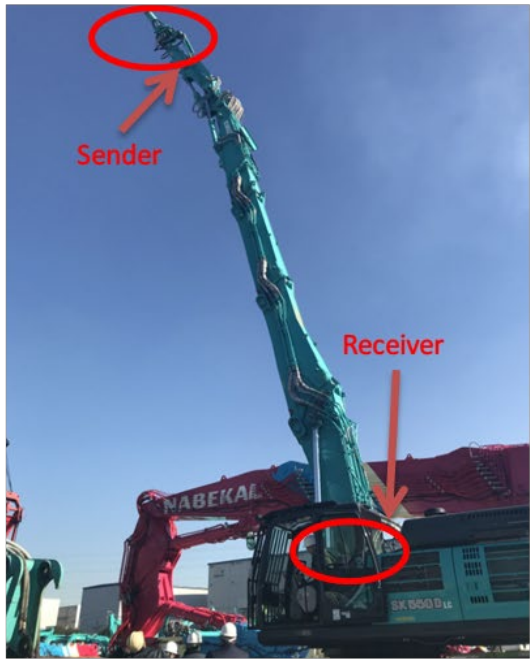

Highest

Figure 15. Postures of excavator (SK470-10).

Dismantling work

used as a sender (communication distances: 10 and $30 \mathrm{~m}$ ) and an in-vehicle computer housing an Intel Atom E3950 (Apollo Lake) processor made by Interface [19] was used as a receiver. We evaluated the relationships among distance between sender and receiver, RSSI, and communication establishment time $\left(T_{c}\right)$. Note that the evaluations described in this section focused only on $T_{c}$ because the communication success percentage was either 0 or $100 \%$. When $P_{s}$ was $0 \%$, the result of $T_{c}$ was NG. In the evaluation, we used an application program to receive

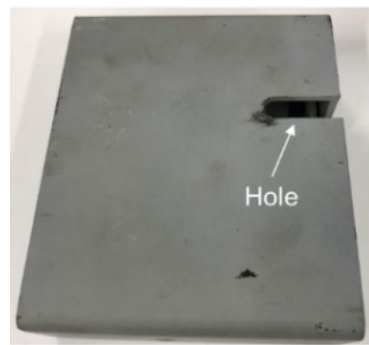

Appearance

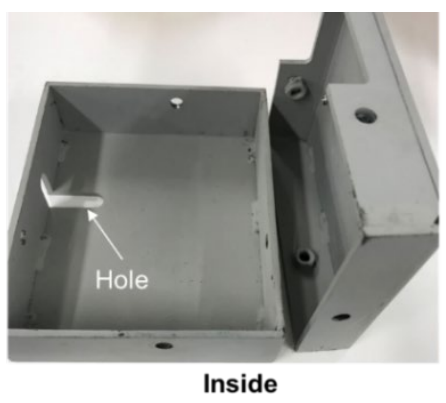

Figure 14. Bracket for beacon. The BLE module was encased in this bracket. The distance between the hole and BLE antenna changed according to the orientation of the BLE module.
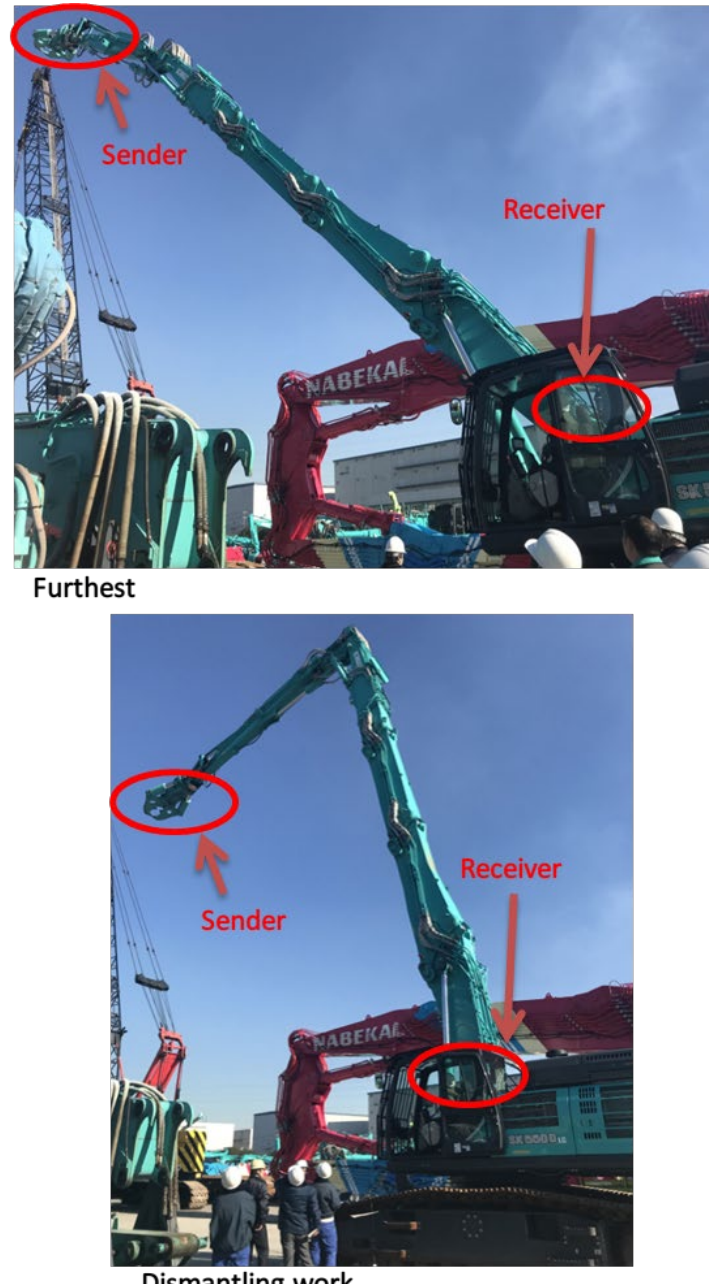


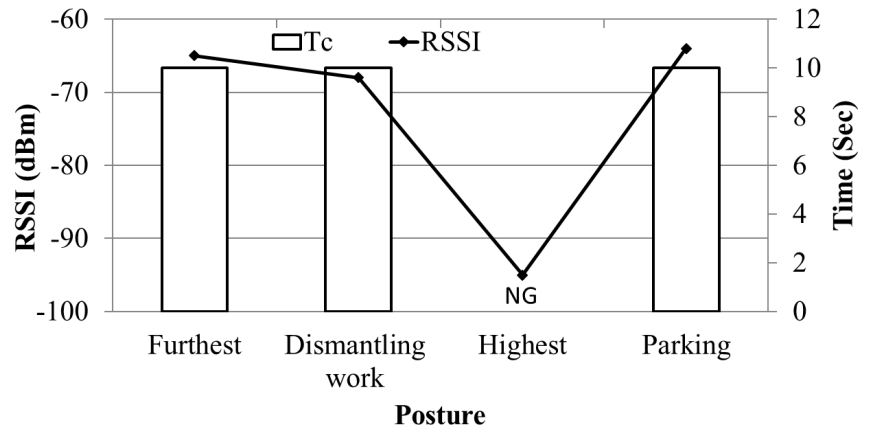

Figure 16. $T_{c}$ and RSSI result for SK470-10 (Beacon communication distance of $10 \mathrm{~m}$; installation was correct).

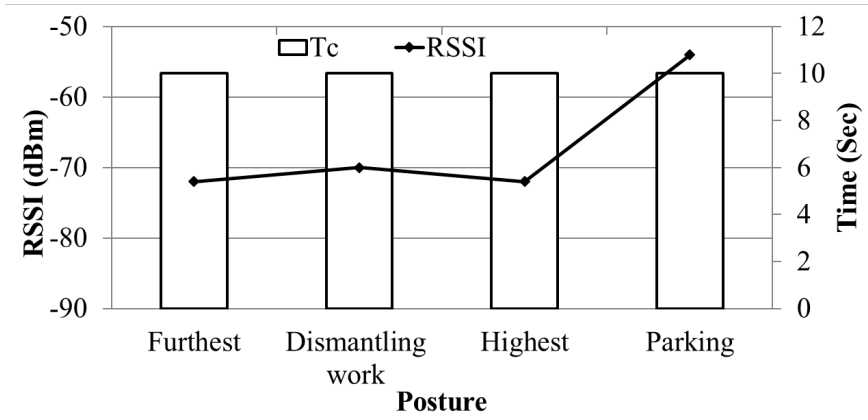

Figure 17. $T_{c}$ and RSSI results for SK470-10 (Beacon communication distance of $30 \mathrm{~m}$; installation was correct).

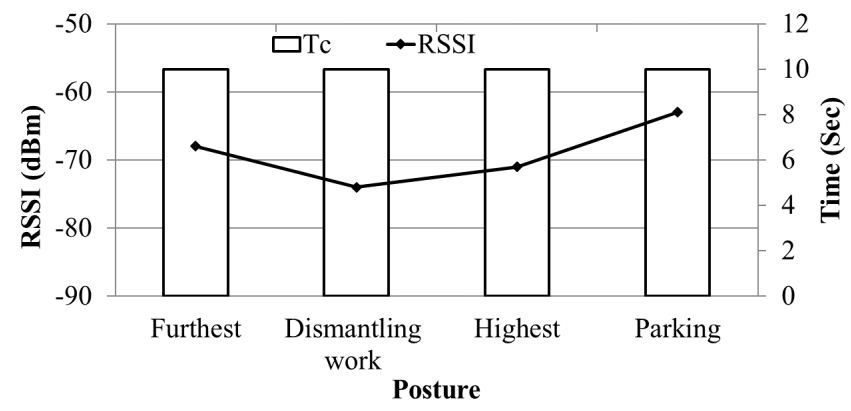

Figure 18. Results of $T_{c}$ and RSSI for SK470-10

(Beacon's communication distance was $30 \mathrm{~m}$, installation is upside down).

the signal produced by a beacon module. Unlike the system used in Section 3.1, the application of the receiver took approximately $10 \mathrm{~s}$ to display results; therefore, when a signal was successfully received, the $T_{c}$ used as the evaluation time was considered to be $10 \mathrm{~s}$. That means that the results were the same when the connection was established in the first attempt $\left(\begin{array}{ll}10 & \mathrm{~s}\end{array}\right)$. The total measurement time was $60 \mathrm{~s}$.

In the evaluations, we attached the beacon module to several excavators. Specifically, we used an SK135-5 [20], an SK470-10 [21], and an SK210DLC-10 [22], which are examples of 13-, 45-, and 20-ton class excavator, respectively. The latter is used for metal recycling. In the evaluation, the module was attached to both the SK135-5 and SK470-10 via a nibbler used for building demolition. For the SK210DLC-10, the sender module was positioned

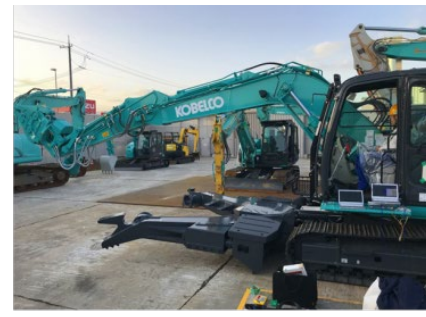

Furthest
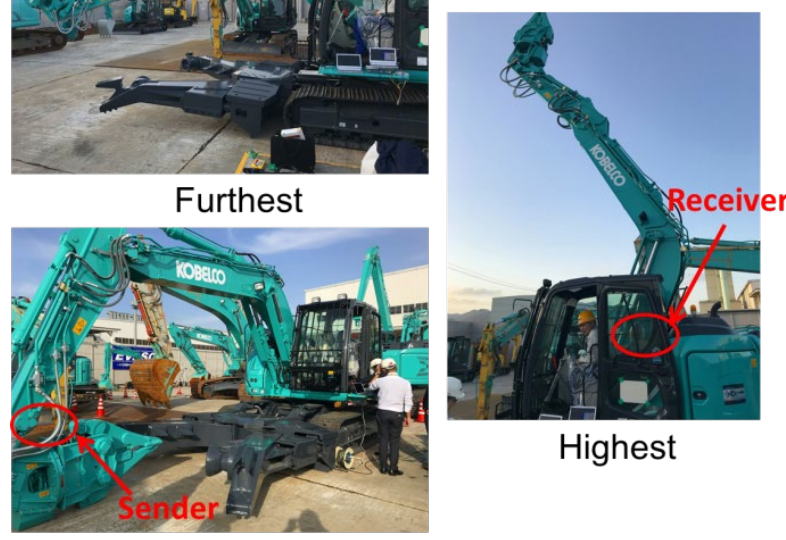

Highest

Parking

Figure 19. Postures of excavator (SK135-5).

on the excavator's magnetic attachment (Fig. 11); in all three cases, the receiver's antenna was placed in the cabin (Fig. 12). The utilisation of the $2.4-\mathrm{GHz}$ band in the evaluation area was measured by a Wi-Fi analyser and is shown in Fig. 13. As shown in the figure, the signal strengths of other wireless communications sources were not large. The sender was placed in a bracket (Fig. 14) with an opening for transmission of radio waves. The hole was set near the BLE antenna. If the module were mounted upside down, the antenna would be further from the radiation hole; therefore, we evaluated the two installation cases. We changed the posture of the excavator (Fig. 15) several times during each evaluation. The different postures were (1) Parking, (2) Highest, (3) Furthest, and (4) Dismantling work. In each posture, the distance between the sender and receiver changed, and we measured the RSSI and $T_{c}$ for each posture over 10 trials and averaged the results. The payload size was 70 bytes and the BLE communication mode was set to connection.

\subsection{Experimental results and discussion by situation}

\subsubsection{Results for SK470-10}

Fig. 16 shows the results when the beacon module's communication distance was set to $10 \mathrm{~m}$. As shown in the figure, the receiver was able to receive the beacon signal in the Parking, Furthest, and Dismantling work postures. Therefore, the $T_{c}$ of each case was $10 \mathrm{~s}$. Moreover, RSSI was approximately $-65 \mathrm{dBm}$. In the Highest posture, however, the beacon signal was too weak and could not be received. The RSSI was approximately $-95 \mathrm{dBm}$. From these results, when the excavator was in the Highest posture, communication could not be established at a beacon module communication distance of $10 \mathrm{~m}$,. Fig. 17 shows the results at a communication distance of $30 \mathrm{~m}$. As shown in the figure, unlike the previous 
communication distance setting, the receiver and sender modules were able to establish communication in all postures. Additionally, the measured RSSI was approximately $-55 \mathrm{dBm}$ in the Parking posture as a result of the short distance between the sender and the receiver. From the results displayed in Figs. 16 and. 17, it was established that a $30-\mathrm{m}$ communication distance setting of the beacon module was required to establish communication in all postures.

Figs. 16 and 17 show the results obtained when the beacon module was correctly installed in the bracket. Fig. 18 plots the results when the beacon module was installed upside down in the bracket with the communication distance of the beacon set to $30 \mathrm{~m}$. It is seen from Fig. 18 that communication could still be established even with an upside down installation configuration. In all postures, the RSSI was at least $-75 \mathrm{dBm}$. A comparison of Figs. 17 and 18 shows that the RSSI was nearly the same under both installation conditions. Thus, if the module's communication distance was set to $30 \mathrm{~m}$, the installation orientation of the beacon module did not significantly affect the BLE communication performance.

From these results, we can conclude that BLE

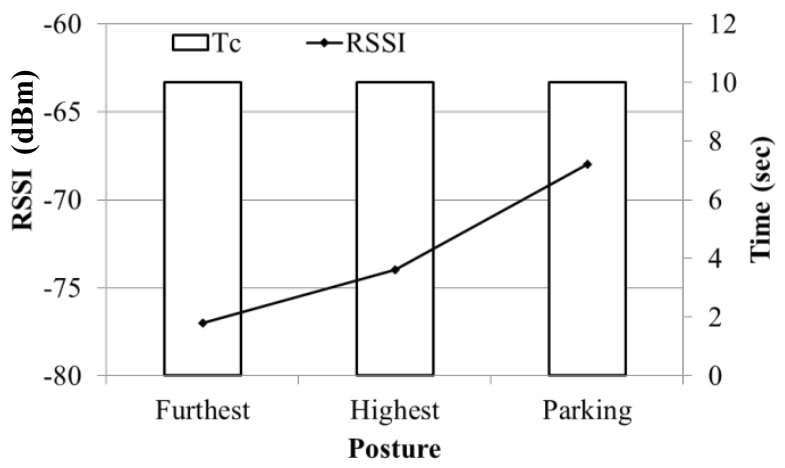

Figure 20. Results of $T_{c}$ and RSSI for SK135-5 (Beacon's communication distance was $10 \mathrm{~m}$, installation was correct).

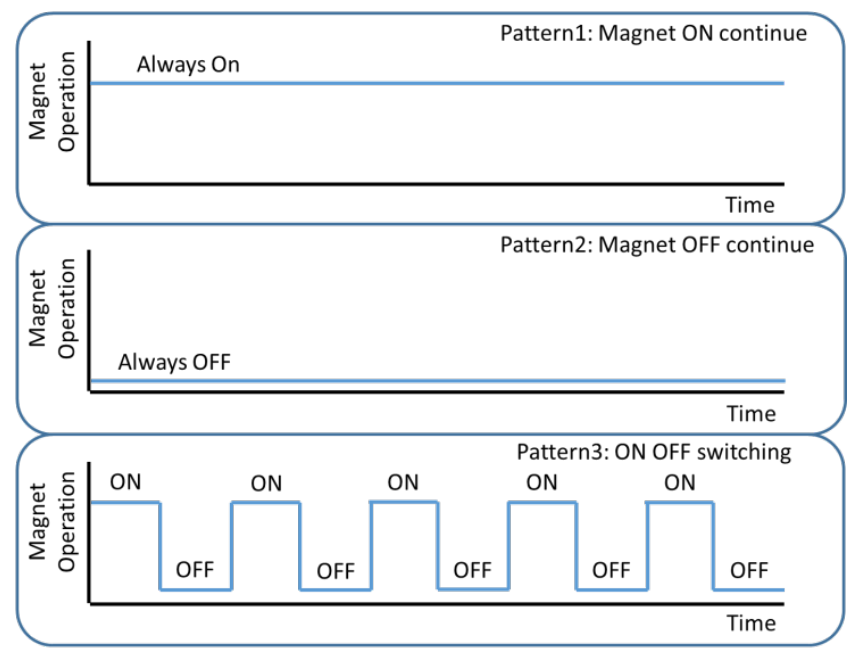

Figure 21. Magnet operational patterns. communication can be used for internal and external communication by 45 -ton class excavators. The following subsection shows the results for the smaller excavators.

\subsubsection{Results for SK135-5}

This subsection shows the results obtained using the SK135-5, a 13-ton class excavator. This machine is used in urban construction areas because of its relatively compact size.

The same items used to evaluate the SK470-10 were used to evaluate the SK135-5. Note that the utilisation trend for the $2.4-\mathrm{GHz}$ band in the evaluation field was the same as that shown in Fig. 13. Fig. 19 shows the postures of the SK130-5. We evaluated only three posturesFurthest, Highest, and Parking - as the results for the Dismantling posture were nearly the same as those for Highest. Fig. 20 shows the relationships among posture, RSSI, and $T_{c}$. In this case, the distance under the Furthest posture was larger than that under Highest, which in turn was larger than that under Parking. From these results, the distance between the sender and receiver increased when the RSSI decreased, a trend seen in the evaluation results. The minimum RSSI (obtained in the Furthest case) was approximately $-77 \mathrm{dBm}$. Even at the minimum RSSI,
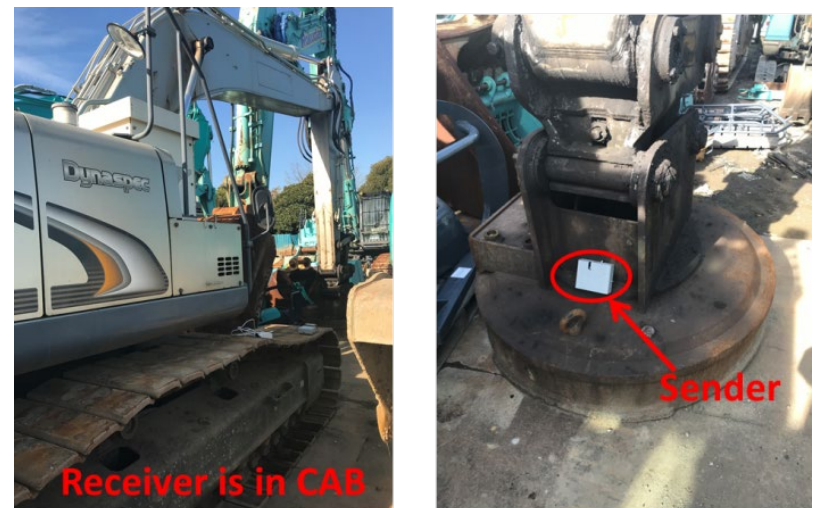

Figure 22. Posture of excavator (SK210DLC-10).

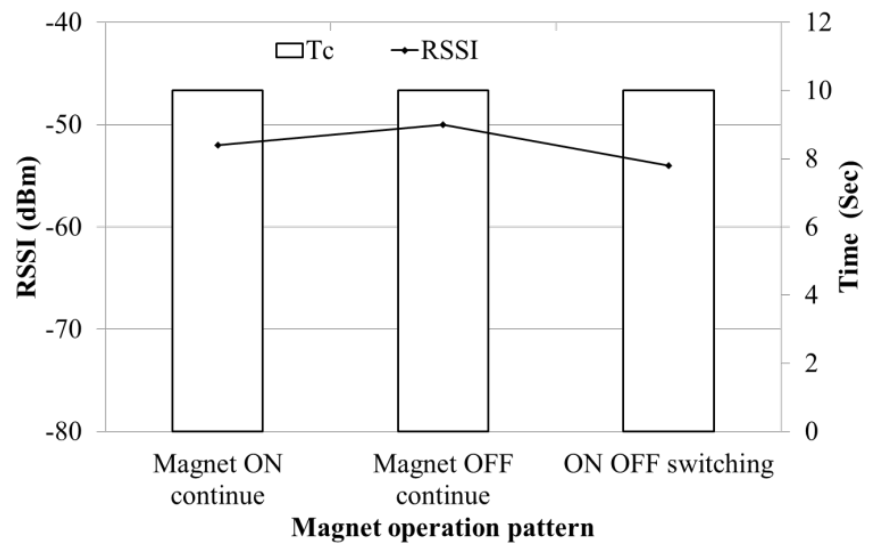

Figure 23. $T_{c}$ and RSSI results for SK210DLC-10 (Beacon communication distance was $10 \mathrm{~m}$; installation was correct). 
however, communication could be established between the sender and receiver. Thus, we can conclude that BLE communication can be applied in the use of 13-ton class excavators.

\subsubsection{Results for SK-210DLC-10}

This subsection shows the results obtained for the SK210DLC-10, a 20-ton class excavator. Excavators of this class can be equipped with a magnetic attachment for gathering metal and scrap, making them useful in metal recycling factories. In these analyses, therefore, we evaluated the BLE communication characteristics with the magnetic field effect taken into consideration under three magnet operational patterns (Fig. 21):

- Magnet ON continuously;

- Magnet OFF continuously;

- ON/OFF switching (changing every $3 \mathrm{~s}$ ).

In the evaluations, we focused on the results for $T_{c}$ and RSSI according to under different magnet operational patterns. Fig. 22 shows the posture of the SK210DLC-10, which in this case remained constant for all tests. In the evaluations, the sender was fixed to the magnet attachment and the receiver was located in the cabin. Note that the trend of utilisation for the $2.4-\mathrm{GHz}$ band in the evaluation field was the same as that shown in Fig. 13.

Fig. 23 plots the relationship among RSSI, $T_{c}$, and the magnet operational pattern. As shown in the figure, the operational did not have a significant effect on the RSSI. Thus, communication could be established normally. Note that communication could be carried out continuously for periods of $60 \mathrm{~s}$. From these results, we can conclude that BLE communication can be applied to a wide range of construction machinery, thus verifying the applicability of BLE to i-construction.

\subsection{Limitations and constraints}

This study includes some limitations and constraints as follows:

- Evaluation system

In this study, we used simple evaluation system.

Communication module is only sending/receiving the BLE signal, and we do not implement any applications that considering communication QoS/QoE, and control of construction machines. Therefore, the functions of the system are simple, only sending the signal by BLE beacon and showing the result by tablet PC (monitor). Our evaluation showed the results with the above assumptions.

- Evaluation metrics

In the evaluation, we showed the characteristics of BLE considering construction machines using RSSI, the communication establishment time, and communication success percentage. This study is the first step for wireless communication characteristics considering construction machines. Therefore, we use simple metric. In future, we will evaluate the communication characteristics using other metric. After implementing applications such as iconstruction systems, especially, we will evaluate the usability of it using the metrics proposed by [23]. Also, we will show the analysis of i-construction systems using Use Cases Tool such as UML diagram.

\section{Conclusion}

In this study, BLE communication was characterised and demonstrated using several real pieces of construction machinery. Specifically, we focused on the applicability of BLE for i-construction. In the evaluation, a beacon module that can communicate using BLE 4.2 was used. From the experimental evaluations, we confirmed the following results:

- We demonstrated the characteristics of communication in both a residential area and at a construction site.

- We quantitatively clarified the relationship among the communication distance, RSSI, and the time to establish device communication.

- We quantitatively demonstrated the effect of length of payload on communication.

- We confirmed the communication characteristics of the beacon module using 45-, 13-, and 20-ton class excavators.

The results of this study confirmed the applicability of BLE for i-construction. We recommend BLE for iconstruction applications because of the payload size that can be communicated and the communication connectivity that does not so affect the size of the construction machines and the type of attachments for construction machines.

Future work will include more detailed evaluations carried out in environments with multiple senders to obtain a better understanding of loss rate and communication delay. We will also propose specific applications/systems of i-construction using BLE. After proposing i-construction systems, we will evaluate the usability of it. Moreover, we will analyze the systems using Use Cases tools.

\section{Acknowledgements.}

We thank anonymous reviewers for carefully reading our paper. Moreover, we would like to thank Editage (www.editage.jp) for English language editing.

\section{References}


[1] K. Endo, R. Hamamoto, H. Tanaka, K. Yamashita, M. Kagoshima, H. Togo, K. Mizukoshi, H. Kawaguchi and Y. Kishino, "A basic study on communication characteristics by bluetooth low energy for i-construction," Proc. ASON 2019, pp.47-50, 2019.

[2] Ministry of Land, Infrastructure, Transport and Tourism of Japan available at http://www .mlit.go.jp/tec/iconstruction/index.html, cited 2020.

[3] D. Kim, K.W. Oh, D. Hong, J.-H. Park, and S.-H. Hong, "Remote control of excavator with designed haptic device," Proc. Int. Conf. on Control, Automation and Systems, pp.1830-1834, 2008.

[4] Y. Shimano, Y. Kami, and K. Shimokaze, "Development of PC210LCi-10/PC200i-10 machine control hydraulic excavator," Komatsu technical report, vol.60, no.167, 7 pages, 2014.

[5] D.B. Durocher and G.R. Feldmeier, "Predictive versus preventive maintenance," IEEE Industry Applications Magazine, vol.10, no.5, pp.12-21, 2004.

[6] Bluetooth SIG, avaialble at https://www.bluetooth.com, cited 2020.

[7] K.V.S.S.S.S. Sairam, N. Gunasekaran, and S.R. Redd, "Bluetooth in wireless communication," IEEE Communications Magazine, vol. 40, no. 6, pp. 90-96, 2002.

[8] W. Bronzi, R. Frank, G. Castignani, and T. Engel, "Bluetooth low energy for inter-vehicular communications," Proc. IEEE VNC 2014, pp. 215-221, 2014.

[9] H. Asano and K. Mase, "Connectivity experiments in a Bluetooth-based multi hop networks," Proc. WPMC 2003,

MA7-4, 2003.

[10] M. Boric, A.F. Vilas, and R.P.D. Redondo, "BLE broadcasting impact in a real network environment," Proc. ICC 2017, 2017.

[11] M. Fainberg and D. Goodman, "Analysis of the interference between IEEE $802.11 \mathrm{~b}$ and Bluetooth systems,' Proc. IEEE VTC Fall 2001, vol. 2, pp. 967-971, 2001.

[12] R. de Francisco, L. Huang, G. Dolmans and H. de Groot, "Coexistence of ZigBee wireless sensor networks and Bluetooth inside a vehicle," Proc. IEEE PIMRC 2009, pp. 2700-2704, 2009.

[13] H. Jin, C. Xu, and K. Lyons, "Corona:Positioning adjacent device with asymmetric bluetooth low energy RSSI distribution," Proc. Annual ACM Symp. UIST 2015, pp.175-179, 2015

[14] S.P. Wijetunge, U.S. Wijetunge, G.R.V. Peiris, C.S. Aluthgedara, and A.T.L.K. Samarasinghe, "Design and implementation of a bluetooth based general purpose controlling module," Proc. Int. Conf. on ICAFS 2008, pp.206-211, 2008

[15] C.-L. Wang and J.-C. Renn, " Study on the motion control of pneumatic actuator via wireless Bluetooth communication," Proc. IEEE ICASI 2018, pp.601-604, 2018.

[16] P. YuHai, "The research of wireless manufacturing network based on bluetooth technology," Proc. Int. Symp. on ISE 2008, pp.457-460, 2008.

[17] T. Golubeva and S. Konshin, "The research of possibility of sharing use of wireless and mobile technologies for organizing the radio channels of operation control system of earthmoving and construction machines," Proc. Int. Conf. INCoS 2016, pp.9-14, 2016.
[18] Wi-Fi analyzer, available at https://play.google.com/store/apps/details?id=com.farproc. wifi.analyzer, cited 2020.

[19] Interface Corporation, available at http://www.interface.co.jp/sfc_etc/sfc_e3950_f.asp, cited 2020.

[20] The specification of SK135-5, available at https://www.kobelco-kenki.co.jp/products/shovel /SK135SR-5.html, cited 2020.

[21] The specification of SK470-10, available at https://www.kobelco-kenki.co.jp/products/shovel/SK47010.html, cited 2020 .

[22] The specification of SK210DLC-10 lifting magnet, available at https://www.kobelcokenki.co.jp/products/spl/mlgrpr00000013qzatt/Metal_recycle_machine.pdf, cited 2020.

[23] A.P. Singun, "Usability metrics for a web-based test blueprint system" Journal of Engineering and Applied Sciences, vol.12, pp.6898-6903, 2017.

\section{Appendix A. Pseudocode of the evaluation system}

This section shows the algorithms of our evaluation system by the pseudocode.

\section{Algorithm 1. Pseudocode of the BLE signal sender for Section 3 and Section 4.}

REQUIRE: TSEND $>0 / /$ TSND is sending interval

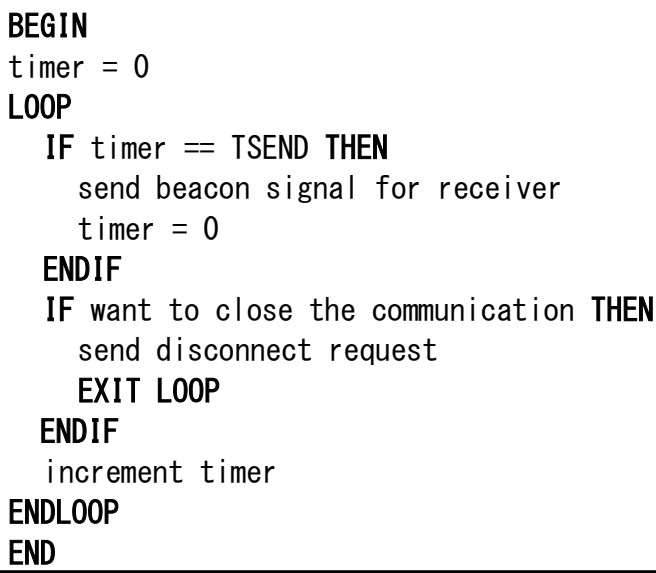
for Section 3.

REQUIRE: TWAIT $>0 / /$ TWAIT is waiting time $/ /$ for timeout to receive //the signal

BEGIN

timer $=0$

LOOP 

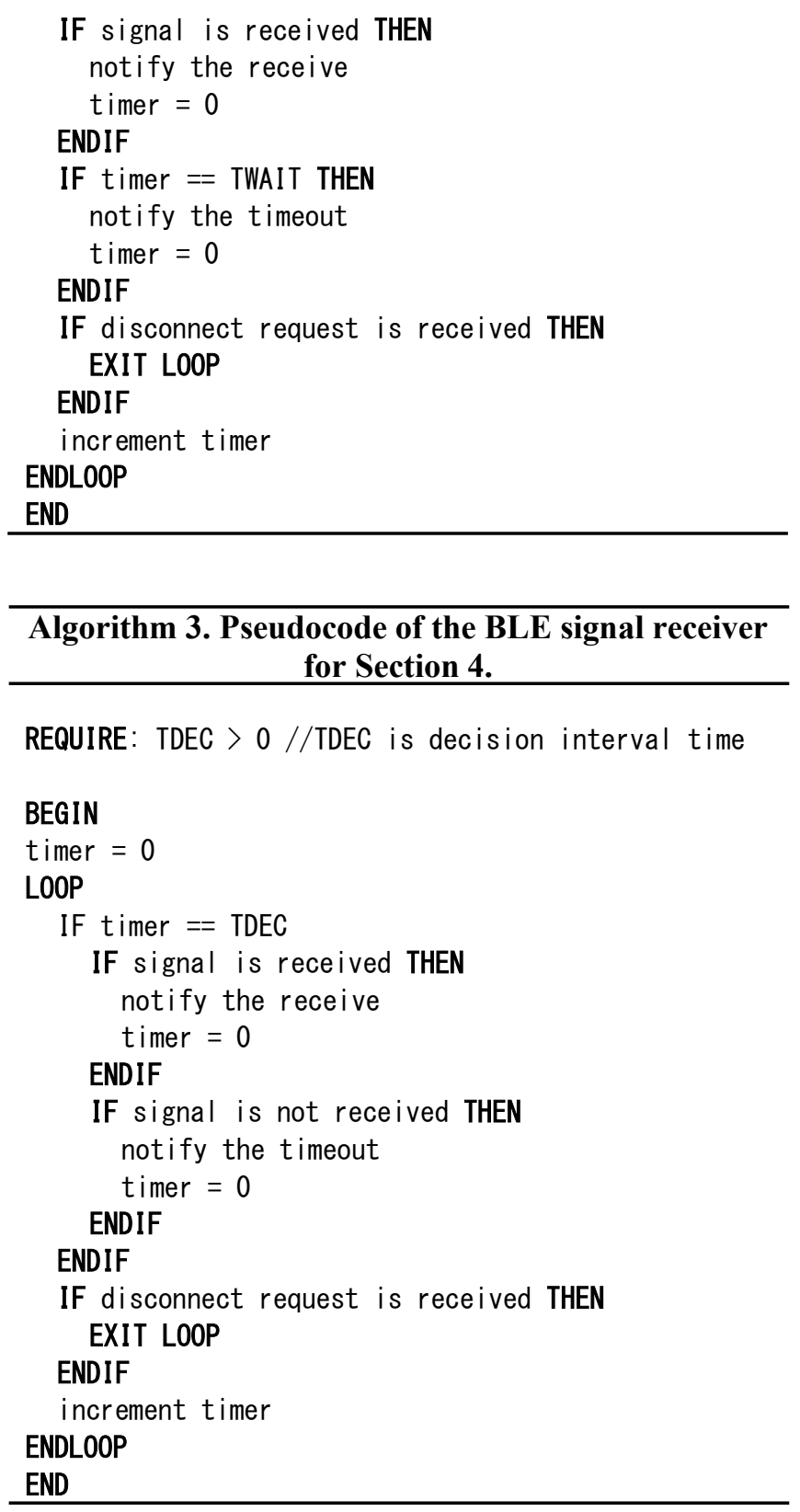

In Section 3, we focused the relationship between the connection and the distance between the sender and the receiver. Thus, we do not set the decision interval. On the other hand, in Section 4, we focused the maximum time to reach the signal. In other words, the motivation of the evaluation of Section 4 was to check that the signal (data) is received within the prescribed time. Therefore, we changed the receiver's algorithm in each evaluation. 